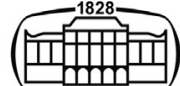

AKADÉMIAI KIADÓ

\section{Acta Veterinaria}

Hungarica

69 (2021) 3, 249-255

DOI:

$10.1556 / 004.2021 .00033$

(c) 2021 The Author(s)

\title{
Genetic parameters of gestation length trait in dromedary camels (Camelus dromedarius)
}

\author{
SZABOLCS BENE $^{1 *}$ @, FERENC SZABÓ $^{2}$, PÉTER J. POLGÁR ${ }^{1}$, \\ JUDIT JUHÁSZ ${ }^{3}$ and PÉTER NAGY ${ }^{3}$ \\ ${ }^{1}$ Institute of Animal Sciences, Georgikon Campus, Hungarian University of Agriculture and Life \\ Sciences, Deák F. u. 16, H-8360 Keszthely, Hungary \\ ${ }^{2}$ Department of Animal Sciences, Faculty of Agricultural and Food Sciences, Széchenyi István \\ University, Mosonmagyaróvár, Hungary \\ ${ }^{3}$ Emirates Industry for Camel Milk and Products, Dubai, United Arab Emirates
}

Received: 21 April 2021 • Accepted: 26 August 2021

Published online: 3 September 2021

\section{RESEARCH ARTICLE}

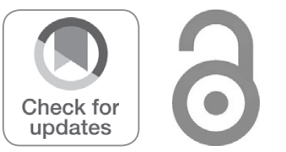

* Corresponding author. Tel.: +36 (83) 545398.

E-mail: bene.szabolcs.albin@uni-mate. hu

\begin{abstract}
Gestation length (GL) data of dromedary camels were analysed for the period from 2007 to 2018 . The database of the largest dairy camel herds (Dubai, United Arab Emirates) was used in this study. The data of 4,084 camels included in the assessment were classified into six ecotypes (Emirati, Emirati cross, Black, Pakistani, Saudi-Sudanese and Saudi cross). The aim of the study was to describe the heritability of GL of camels and the breeding value (BV) of sires for this trait. The genetic parameters of GL were estimated by the General Linear Model method and two Best Linear Unbiased Prediction (BLUP) animal models as well. The mean $( \pm \mathrm{SE})$ of GL of camels was $384.3 \pm 0.2$ days. The direct heritability of GL $(0.26 \pm 0.06-0.36 \pm 0.08)$ was higher than the maternal heritability $(0.00 \pm 0.05-0.13 \pm 0.06)$ obtained. The maternal permanent environmental effect $(0.15 \pm 0.05)$ was similar to the results estimated previously in dromedary camel, but higher than the data reported by relevant sources in other species. Based on the results of this study it can be concluded that the GL of dromedary camels is a species-specific value similar to that in cattle, which is less affected by the maternal influence. Considerable differences (16 days) exist among male dromedaries in their BV for the GL trait.
\end{abstract}

\section{KEYWORDS}

dromedary camel, gestation length, BLUP, heritability, breeding value

\section{INTRODUCTION AND REVIEW OF THE LITERATURE}

Gestation length (GL) is a basic reproductive trait in mammals with a species-specific standard value and low coefficient of variation (1.4\%-3.25\%; Heck et al., 2018) that also shows little difference among breeds. Thus, it is generally accepted that pregnancy length is less affected by environmental factors, so the heritability of this trait is typically moderate to medium or good (Bene et al., 2014). This might be the reason why GL in different farm animals (e.g. horses and cattle) has been studied recently mainly from the veterinary point of view for animal health aspects (e.g. the correlation between GL and bone development; Haywood et al., 2017), as well as for selection criteria. At the same time, research on the animal breeding or genetic aspects of this trait is rather limited in domestic animals (Rodrigues et al., 2020).

The length of pregnancy in dromedary camels, like in other species, has a low coefficient of variation (2.9\%); however, it still varies within a wide range (from 333 to 422 days) due to the relatively long gestation period (Nagy and Juhász, 2019). Moreover, there are considerable differences in the mean value of GL among various studies. Metha et al. (1962) reported $389 \pm 28$ days for GL for Bikaner camels in India. Ram et al. (1977) published longer GL (404 \pm 5 days) also for Bikaner camels than the previous authors. In contrast, Yagil and Etzion 
(1984) found lower values (345-360 days) for the GL of camels of different genotypes. Musa et al. (2006) observed a GL of $370 \pm 19$ days in a small camel population in Western Sudan. According to Musa and Abu Sineina (1976) and AlBisher (1998), the GL of camels ranged from 373 to 393 days. Almutairi et al. (2010) found that the GL of Saudi camels was $378 \pm 9$ days based on 414 calving data.

There are few studies in the literature on factors influencing the GL of camels. According to Almutairi et al. (2010), the GL of camels calving in autumn and winter was 6 days longer compared to those that delivered their calf during the spring and summer months. The age of the camel and the sex of the calf had no effect on GL. Earlier, Sharma and Vyas (1971) similarly demonstrated the effect of the month of calving in Bikaner camels and also confirmed the effect of the sire on GL. Based on data from the previous sources, Al Eknah (2000) concluded that the GL of dromedary camels is mostly affected by the age of the camel at pregnancy (Novoa, 1970), the sex of the calf (Agarwal et al., 1987), the level of feeding (Yagil and Etzion, 1984), and the month of conception (Elias et al., 1991). Recently, Nagy and Juhász (2019) have described the relationship among numerous variation factors that influence GL in dromedaries. They have demonstrated that environmental and physiological factors had the strongest relative effect (42.3\%) on the variation, while factors of genetic origin (female camel, sire) had a lower, but still considerable influence $(17.7 \%)$ on GL in this species.

No information was found in the literature on the genetic parameters, heritability $\left(\mathrm{h}^{2}\right)$ of GL and breeding value (BV) of the animals based on this trait in dromedary camels. However, for other species, such as cattle or horses, a small number of sources have reported data on the heritability of this trait (Table 1). In cattle, Bourdon and Brinks (1982),

Table 1. Genetic parameters of gestation length trait in literature sources

\begin{tabular}{lccccc}
\hline & & \multicolumn{5}{c}{ Gestation length } \\
\cline { 3 - 6 } Source & Species & $\mathrm{h}_{\mathrm{d}}{ }_{\mathrm{d}}$ & $\mathrm{h}_{\mathrm{m}}{ }_{\mathrm{m}}$ & $\mathrm{r}_{\mathrm{dm}}$ & $\mathrm{c}^{2}$ \\
\hline Bourdon and & cattle & $0.36-$ & - & - & - \\
$\quad$ Brinks (1982) & & 0.37 & & & \\
Wray et al. (1987) & cattle & 0.37 & - & - & - \\
Hansen et al. (2004) & cattle & 0.42 & 0.07 & - & - \\
Crews (2006) & cattle & 0.64 & 0.07 & -0.37 & 0.03 \\
Valera et al. (2006) & horse & 0.21 & - & - & 0.14 \\
Mujibi and Crews & cattle & 0.62 & 0.10 & - & - \\
$\quad(2009)$ & & & & & \\
Langlois and Blouin & horse & $0.09-$ & $0.08-$ & 0.05 & $0.03-$ \\
$\quad(2012)$ & & 0.12 & 0.16 & & 0.06 \\
Christmann et al. & horse & 0.03 & 0.23 & - & - \\
$\quad(2017)$ & & & & & \\
Ewert et al. (2018) & cattle & - & 0.17 & - & - \\
Rodrigues et al. & horse & 0.19 & 0.39 & 0.02 & 0.01 \\
$\quad(2020)$ & & & & & \\
\hline
\end{tabular}

$\mathrm{h}_{\mathrm{d}}^{2}=$ direct heritability; $\mathrm{h}_{\mathrm{m}}^{2}=$ maternal heritability; $\mathrm{r}_{\mathrm{dm}}=$ directmaternal genetic correlation; $c^{2}=$ the ratio of the permanent environmental variance to the phenotypic variance.
Wray et al. (1987) and Crews (2006) found the direct heritability $\left(\mathrm{h}_{\mathrm{d}}^{2}\right)$ of GL to be between 0.36 and 0.64 . In the equine species, Valera et al. (2006) and Langlois and Blouin (2012) reported $0.09-0.21$ values for the $\mathrm{h}^{2}{ }_{\mathrm{d}}$ of this trait. Christmann et al. (2017) and Rodrigues et al. (2020) found values of $0.23-0.39$ for maternal heritability $\left(\mathrm{h}^{2}{ }_{\mathrm{m}}\right)$ of GL in Hanoverian and Lusitano horses, respectively.

The objectives of this study were (1) to determine some genetic parameters, especially the heritability of GL in dromedary camel, (2) to estimate the BV of male dromedaries for this trait, and (3) to compare the results obtained by different GLM and BLUP models using the world's largest available dromedary camel dataset. Based on our previous results (Nagy and Juhász, 2019), we hypothesised that GL in dromedary camels is primarily influenced by environmental factors, thus the $\mathrm{h}^{2}$ of this trait may be low in camels, as opposed to other farm animal species.

\section{MATERIALS AND METHODS}

The present study is a continuation of our earlier work (Bene et al., 2020) on dromedary camels. Due to the similarity between the database and methods used, some details of the Materials and Methods described in our previous publication will not be repeated here.

\section{Location of the study, number of animals}

The study was conducted over 11 breeding seasons from 2007 through 2018 at the premises of Emirates Industry for Camel Milk and Products (EICMP), the world's first largescale camel dairy farm that is located in Dubai, United Arab Emirates. During this period, a total of 58 male (bull) and 2,087 female (dam) dromedaries were included into the breeding programme and 4,084 calves (progeny) were delivered on the farm (Table 2). Further details on farm management have been described previously (Nagy et al., 2013; Bene et al., 2020). Data were included only for deliveries between 344 and 420 days of gestation (range 76 days), and outliers below and above this range were excluded from the analysis.

Like in our previous work, camels were categorised into 6 ecotypes (Emirati, Emirati cross, Black, Pakistani, Saudi/ Sudanese and Saudi cross) based on geographical origin,

Table 2. Composition of the examined population

\begin{tabular}{lc}
\hline Generation groups & Number of animals \\
\hline Calves born recorded & 4,084 \\
Sires & 58 \\
Dams & 2,087 \\
Paternal grandsires & 7 \\
Maternal grandsires & 22 \\
Grandsires, total & 29 \\
Paternal granddams & 9 \\
Maternal granddams & 269 \\
Granddams, total & 278 \\
\hline
\end{tabular}


colour, appearance, and body conformation (Abdallah and Faye, 2012; Fábri, 2018).

\section{Gestation length}

The gestation length trait was considered as a character of the calf, since during this time the calf is developing in the uterus. While constructing the pedigree model, the paternal and maternal origins of calves were used. Thus, the studied and presented breeding value data are referred to the sire of calves.

The effects on the manifestation of the GL were interpreted as follows: Direct effect: the inherited growth and development length of the calf in the uterus. The inheritance of growth comes from the sire and the dam in half. Maternal genetic effect: an inherited part of a female camel's calfrearing ability, including mitochondrial effects. Maternal permanent environmental effect (PE): the uterus is an environment for the calf. Therefore, the maternal environment effect is the sum of the effects that the uterus, as an environment, has on the developing progeny (e.g. through its size and how it supplies the progeny with blood and other important substances and how it secures fetal movements, etc.).

\section{Estimation of genetic parameters}

The Kolmogorov-Smirnov test was used to check the normal distribution of the GL in the database. The homogeneity of variances was examined by Levene's test.

To estimate the genetic parameters of GL three different models (Szőke and Komlósi, 2000) were designed: one was GLM (Type III) model and two BLUP (Henderson, 1975) animal models. The details of the three models are summarised in Table 3.

Table 3. Models used for the estimation of genetic parameters for the gestation length trait in dromedary camels

\begin{tabular}{lcccc}
\hline Used models & Classes & GLM & BLUP1 & BLUP2 \\
\hline Random effects & & & & \\
- sire (male camel) & 58 & + & - & - \\
- animal (newborn calf) & 4,084 & - & + & + \\
- maternal genetic effect & 2,087 & - & + & + \\
- maternal permanent & 2,087 & - & - & + \\
$\quad$ environmental effect & & & & \\
Fixed effects & & & & \\
- ecotype of dam & 6 & + & + & + \\
- parity of dam & 5 & + & + & + \\
- breeding season & 11 & + & + & + \\
- month of mating & 9 & + & + & + \\
- sex of calf & 2 & + & + & + \\
Pedigree matrix & & & & \\
- animal (newborn calf) & & - & + & + \\
- sire (male camel) & & - & + & + \\
- dam (cow, female camel) & & - & + & + \\
- full sibs, half sibs & & - & + & + \\
- grandparents & & - & + & + \\
\hline
\end{tabular}

$+/-=$ the model includes/does not include this effect; GLM = General Linear Model; BLUP $=$ Best Linear Unbiased Prediction.
The examined fix (environmental) factors for all models were as follows: ecotype of dam, parities of dam, breeding season, month of mating, and the sex of the calf (Van Vleck et al., 1996; Lee et al., 1997). Covariant was not included into the models.

The calculation procedure of the components in the case of GLM method was described in our previous work (Bene, 2013) and therefore it is not detailed here. The model was constructed as follows:

GLM : $\widehat{\mathrm{y}}_{\mathrm{ijklmn}}=\mu+\mathrm{S}_{\mathrm{i}}+\mathrm{E}_{\mathrm{j}}+\mathrm{P}_{\mathrm{k}}+\mathrm{Y}_{\mathrm{l}}+\mathrm{M}_{\mathrm{m}}+\mathrm{I}_{\mathrm{n}}+\mathrm{e}_{\mathrm{ijklmn}}$

where: $Y_{i j k l m n}=G L$ of calf from ' $i$ ' sire, in ' $j$ ' ecotype, in ' $k$ ' parity, in ' $\mathrm{l}$ ' season and ' $\mathrm{m}$ ' month and ' $\mathrm{n}$ ' sex; $\mu=$ overall mean value; $S_{i}=$ random effect of sire; $E_{j}=$ fix effect of ecotype; $\mathrm{P}_{\mathrm{k}}=$ fix effect of parity of dam; $\mathrm{Y}_{1}=$ fix effect of season; $M_{m}=$ fix effect of month of mating; $I_{n}=$ fix effect of sex of calf; $\mathrm{e}_{\mathrm{ijklmn}}=$ residual.

Using the BLUP models, two matrices were created. One of these was the database matrix and the other was the pedigree matrix. The pedigree matrix of relatives included pedigree data for full sibs, half sibs, sires, dams, and grandparents. BLUP models contained information for maternal genetic effect (BLUP1 and BLUP2) and maternal permanent environmental effect (only BLUP2) as random effects. The BLUP2 model was similar to that used by Rodrigues et al. (2020) to study the GL of horses. The models were constructed as follows:

$$
\begin{gathered}
\text { BLUP1 : } \widehat{y}=X_{b}+Z_{u}+W_{m}+e \\
\text { BLUP2: } \widehat{y}=X_{b}+Z_{u}+W_{m}+S_{p e}+e
\end{gathered}
$$

where: $\hat{y}=$ vector of observation - GL); $b=$ vector of fixed effects (ecotype, parity of dam, season, month of mating and sex of calf); $\mathrm{u}=$ vector of random effect (animal); $\mathrm{m}=$ vector of maternal genetic effect; pe $=$ vector of maternal permanent environmental effect; $\mathrm{e}=$ vector of random residual effect; $\mathrm{X}=$ matrix of fixed effects; $\mathrm{Z}=$ matrix of random effects; $\mathrm{W}=$ matrix of maternal genetic effect; $\mathrm{S}=$ matrix of maternal permanent environmental effect.

To determine the most suitable model for estimating the parameters, the $\mathrm{e}^{2}$ values and $\log$-likelihood values $(-2 \log \mathrm{L})$ for the three different models were compared (Bouwman et al., 2010; Alves et al., 2018).

A simple Pearson's phenotypic correlation coefficient was calculated between the gestation length and the birth weight (BW) traits.

\section{Estimation of the breeding value of sires}

The breeding value of the dromedary sires for the GL trait was estimated with all three models. In case of the GLM method, BV was considered as the double of the realised progeny difference (RPD), namely $B V=2 \mathrm{RPD}$. The RPD was defined as the difference of the mean value of the GL data of close relatives (progeny, sibs and half-sibs) of a particular dromedary sire and the mean value of the GL data of the contemporary calf group. In the case of BLUP model, the animal model communicated the values of BV directly. 
BVs were estimated only for male dromedaries $(n=18)$ with at least 100 progeny.

\section{Software used}

Variance, covariance, correlation, heritability and breeding values according to the above-mentioned three models were evaluated as described by Willham (1972), Trus and Wilton (1988) and Lee et al. (1997). HARVEY (Harvey, 1990), DFREML (Meyer, 1998) and MTDFREML (Boldman et al., 1993) software packages were used for the estimation.

\section{RESULTS}

The descriptive statistics of GL are shown in Table 4. The mean GL of dromedary camels was 384.3 days $[\mathrm{SE}=0.2$ day, $\mathrm{SD}=10.9$ days, coefficient of variation $(\mathrm{CV})=2.8 \%$ ].

Table 4. Descriptive statistics of gestation length trait of dromedary camels

\begin{tabular}{lc}
\hline Parameters & Gestation length \\
\hline $\mathrm{N}$ & 4,084 \\
Mean (day) & 384.3 \\
Standard error (day) & 0.2 \\
Standard deviation (day) & 10.9 \\
Coefficient of variation (\%) & 2.8 \\
Median (day) & 383.0 \\
Range (day) & 76 \\
Minimum (day) & 344 \\
Maximum (day) & 420 \\
Kolmogorov-Smirnov test $\dagger(P)$ & 0.000 \\
\hline
\end{tabular}

$\dagger$ if $P>0.05$, normal distribution is confirmed.
A significant but loose correlation value $(r=0.14 ; P<0.01)$ was found between the GL and the BW of dromedary camels.

Genetic parameters estimated with the three models (GLM, BLUP1 and BLUP2) are summarised in Table 5. The direct heritability of the GL of dromedary camels was me$\operatorname{dium}\left(\mathrm{h}_{\mathrm{d}}^{2}=0.26 \pm 0.06-0.36 \pm 0.08\right)$. There was considerable difference in the magnitude of the maternal heritability between the two BLUP models. When permanent maternal environmental effects (PE) were not included in the model (BLUP1), the maternal heritability $\left(\mathrm{h}^{2} \mathrm{~m}\right)$ value was $0.13 \pm 0.06$. Direct heritability $\left(\mathrm{h}_{\mathrm{d}}^{2}\right)$ still exceeded maternal heritability $\left(\mathrm{h}_{\mathrm{m}}^{2}\right)$ more than twice. However, when PE was included in the model (BLUP2), the $\mathrm{h}^{2} \mathrm{~m}$ value was much lower $(0.00 \pm 0.05)$. The correlation $\left(r_{d m}\right)$ estimated between direct and maternal genetic effects seemed to be negative and quite loose $(-0.05 \pm 0.26)$ with the BLUP1 model and could not be estimated with BLUP2 model. In addition, because the SE values were too high in the BLUP1 model, the $\mathrm{r}_{\mathrm{dm}}$ values were not reliable and informative. The $c^{2}$ value estimated by the BLUP2 model is fairly high $(0.15 \pm$ $0.05)$ and reliable, which indicates considerable environmental (management, nutrition, season etc.) effects on the GL of dromedary camels.

The BV of dromedary sires for GL estimated by the models used is summarised in Table 6. Breeding values for direct genetic effects estimated by the GLM model were generally lower than the values obtained by the BLUP models. However, these differences did not influence the ranking of the sires by their BV. Notable differences (from -8.48 to 7.87 days; range 16.35 days) in BV for GL could be observed only among sires that are far up and down in the ranking away from the mean value. Because the $\mathrm{h}_{\mathrm{m}}^{2}$ value was very low (especially for the BLUP2 model), the BV for maternal genetic effects was much lower for direct genetic

Table 5. The estimated genetic parameters for the gestation length trait in dromedary camels

\begin{tabular}{|c|c|c|c|}
\hline \multirow[b]{2}{*}{ Parameter } & \multicolumn{3}{|c|}{ Gestation length } \\
\hline & GLM & BLUP1 & BLUP2 \\
\hline$\sigma_{\mathrm{d}}^{2}$ additive direct genetic variance & 25.47 & 27.23 & 28.74 \\
\hline$\sigma_{\mathrm{m}}^{2}$ maternal genetic variance & - & 10.32 & 0.12 \\
\hline$\sigma_{\mathrm{dm}}$ direct maternal genetic covariance & - & -0.80 & -1.58 \\
\hline $\begin{array}{l}\sigma_{\text {pe }}^{2} \text { maternal permanent } \\
\text { environmental effect }\end{array}$ & - & - & 11.56 \\
\hline$\sigma^{2}{ }_{\mathrm{e}}$ residual variance & 71.49 & 41.86 & 39.97 \\
\hline$\sigma_{p}^{2}$ phenotypic variance & 96.96 & 78.61 & 78.81 \\
\hline $\mathrm{h}_{\mathrm{d}}^{2}$ direct heritability & $0.26 \pm 0.06$ & $0.35 \pm 0.08$ & $0.36 \pm 0.08$ \\
\hline $\mathrm{h}_{\mathrm{m}}^{2}$ maternal heritability & - & $0.13 \pm 0.06$ & $0.00 \pm 0.05$ \\
\hline$r_{d m}$ direct-maternal genetic correlation & - & $-0.05 \pm 0.26$ & not estimated \\
\hline $\begin{array}{l}c^{2} \text { the ratio of the permanent } \\
\text { environmental variance to the } \\
\text { phenotypic variance }\end{array}$ & - & - & $0.15 \pm 0.05$ \\
\hline $\begin{array}{l}\mathrm{e}^{2} \text { the ratio of the residual variance to } \\
\text { the phenotypic variance }\end{array}$ & $0.74 \pm 0.05$ & $0.53 \pm 0.06$ & $0.51 \pm 0.06$ \\
\hline$h_{m}^{2}+c^{2}$ & - & - & 0.15 \\
\hline $\mathrm{h}_{\mathrm{T}}^{2}$ total heritability & - & 0.40 & 0.34 \\
\hline$-2 \log L$ & - & $21,476.4$ & $21,468.4$ \\
\hline
\end{tabular}


Table 6. Breeding values of the dromedary camel sires evaluated for the gestation length trait

\begin{tabular}{|c|c|c|c|c|c|c|c|}
\hline \multirow[b]{3}{*}{ Identity number of sire $\S^{\S}$} & \multirow[b]{3}{*}{ Ecotype of sire } & \multirow[b]{3}{*}{$\mathrm{N}$} & \multicolumn{5}{|c|}{ Breeding values for gestation length by methods of estimation (day) } \\
\hline & & & \multirow[b]{2}{*}{ GLM } & \multicolumn{2}{|c|}{ BLUP1 } & \multicolumn{2}{|c|}{ BLUP2 } \\
\hline & & & & direct effect & maternal effect & direct effect & maternal effect \\
\hline 2010 & Emirati & 162 & +4.73 & +7.87 & -0.91 & +7.76 & -0.43 \\
\hline 2020 & Saudi/Sudanese & 178 & +3.03 & +6.11 & +0.64 & +6.07 & -0.33 \\
\hline 2013 & Emirati & 145 & +3.46 & +5.97 & -1.79 & +5.79 & -0.33 \\
\hline 2053 & Saudi/Sudanese & 105 & +1.23 & +2.49 & +0.64 & +2.48 & -0.13 \\
\hline 2021 & Pakistani & 336 & +0.98 & +2.10 & -0.23 & +2.07 & -0.11 \\
\hline 2040 & Emirati cross & 111 & +1.07 & +2.06 & -0.06 & +2.01 & -0.11 \\
\hline 2045 & Emirati cross & 102 & +0.75 & +1.76 & -0.05 & +1.73 & -0.10 \\
\hline 2043 & Emirati cross & 100 & -0.19 & -0.1 & -0.00 & +0.03 & -0.00 \\
\hline 2001 & Saudi/Sudanese & 204 & +0.12 & -0.34 & 1.06 & -0.38 & +0.03 \\
\hline 2026 & Emirati cross & 254 & -0.37 & -0.77 & -0.11 & -0.83 & +0.05 \\
\hline 2028 & Saudi cross & 195 & -0.88 & -0.68 & +0.02 & -0.96 & +0.05 \\
\hline 2011 & Emirati & 134 & -0.76 & -1.70 & +1.33 & -1.78 & +0.10 \\
\hline 2027 & Saudi/Sudanese & 161 & -2.49 & -3.55 & +0.10 & -3.58 & +0.20 \\
\hline 2015 & Saudi/Sudanese & 101 & -1.84 & -3.94 & +2.28 & -3.97 & +0.23 \\
\hline 2000 & Saudi/Sudanese & 262 & -2.14 & -4.52 & -1.98 & -4.69 & +0.24 \\
\hline 2016 & Black & 101 & -3.43 & -5.97 & +1.74 & -6.03 & +0.34 \\
\hline 2017 & Emirati cross & 173 & -3.55 & -6.57 & -0.36 & -6.66 & +0.36 \\
\hline 2004 & Saudi/Sudanese & 237 & -4.39 & -8.35 & +0.51 & -8.48 & +0.47 \\
\hline
\end{tabular}

$\mathrm{N}=$ number of progeny; $\S=$ males sorted by direct breeding value of the BLUP2 model.

effects, and no considerable differences were found among male dromedaries.

\section{DISCUSSION}

The average GL of camels in our study was slightly higher than the mean that had been reported in the literature (Ram et al., 1977; Yagil and Etzion, 1984; Musa et al., 2006). As compared to other large domestic animal species, the GL of dromedary camels was much longer than that of cattle (Hansen et al., 2004; Goyache et al., 2005) or horses (Bos and Van der Mey, 1980; Pérez et al., 2003). The range and standard deviation of GL of camels in our study were similar to those of horses $(\mathrm{SD}=9.26$ days, range $=60$ days; Rodrigues et al., 2020), but they were higher than those found by Hansen et al. (2004) in cattle ( $\mathrm{SD}=5.09$ days, range $=42$ days). However, it is important to note that intrauterine fetal growth rate is slower in camels than in cattle and horses (Nagy and Juhász, 2019).

According to the $\mathrm{e}^{2}$ and $-2 \log \mathrm{L}$ data (Alves et al., 2018), the BLUP1 and BLUP2 models were equally reliable and were more accurate than the GLM model. As the BLUP2 model differentiates between maternal genetic and maternal environmental effects, it seems to be more appropriate for estimating genetic parameters and BV based on GL in this species.

The results of this study for direct heritability values of the GL of dromedary camels could not be compared to previous data, because no relevant information has been found in the literature. As compare to other species (Table 1), the direct heritability of GL in dromedary camels corresponds to the results of Bourdon and Brinks (1982), Wray et al. (1987) and Hansen et al. (2004) in cattle. In contrast, some authors (Langlois and Blouin, 2012; Christmann et al., 2017; Rodrigues et al., 2020) reported lower values of $\mathrm{h}_{\mathrm{d}}^{2}$ in the GL of horses compared to our findings. Some sources (Crews, 2006; Mujibi and Crews, 2009) reported particularly high direct heritability values $\left(\mathrm{h}^{2}{ }_{\mathrm{d}}>0.6\right)$ for the GL of cattle. Like the previous parameter, the maternal heritability of GL of dromedary camels was closer to that seen in cattle (Hansen et al., 2004; Crews, 2006; Mujibi and Crews, 2009). In contrast, Christmann et al. (2017) and Rodrigues et al. (2020) reported higher $\mathrm{h}_{\mathrm{m}}^{2}$ values in horses compared to our results. The $c^{2}$ value of GL of dromedary camels was similar to the finding of Valera et al. (2006) in horses, but it was much higher than that shown by the data from relevant sources (Crews, 2006; Langlois and Blouin, 2012; Rodrigues et al., 2020) for GL in cattle and horses. Differently from our present results, Lee et al. (1997), Carnier et al. (2000) and Phocas and Laloë (2004) reported very low (0.02-0.04) $\mathrm{c}^{2}$ values for weaning weight, calving ease and birth weight traits in beef cattle breeds, respectively. The relationship between direct and maternal genetic effects $\left(r_{\mathrm{dm}}\right)$ on GL shows a tendency similar to the results of Rodrigues et al. (2020) for horses. However, most other studies in cattle on the GL trait (Crews, 2006), the weaning weight trait (Iwaisaki et al., 2005) and the calving ease trait (Cubas et al., 1991) reported stronger correlation between direct and maternal genetic effects compared to our results. In summary, it can be concluded that the dromedary camel's $\mathrm{h}_{\mathrm{d}}^{2}$ of GL was similar to values in cattle but higher than those in horses, the $\mathrm{h}_{\mathrm{m}}^{2}$ of GL was similar to values in cattle but lower than those in horses, the $r_{\mathrm{dm}}$ value was similar to values in horses but lower than those in cattle, and the $c^{2}$ of GL was higher than those in cattle and horses. 
In this study, the genetic parameters of GL of dromedary camels were partially different from those published for BW of camels previously (Bene et al., 2020). In case of GL the $\mathrm{h}_{\mathrm{d}}^{2}$ was higher, the $h^{2}$ m was lower and the $c^{2}$ was slightly lower that those observed for the BW of camels. The total heritability of GL was considerably higher than that observed for BW. This means that the effect of environmental factors was lower in the case of GL than in the case of BW.

Based on the total heritability value of GL in dromedary camels, it can be stated that the genetic background had a $35-40 \%$ share and the environmental factors played a $60-65 \%$ role in the development of the studied trait. This finding is similar to the results of Nagy and Juhász (2019), in which the environmental factors had a $42.3 \%$ share in the GL of dromedary camels. In addition, the maternal permanent environmental effect on GL and BW in dromedary camels was rather high, which again draws attention to the important role played by environmental factors.

According to the work of Heck et al. (2018), it seems that in the highly controlled production animals (for example in cattle) the variability of GL is lower than in the noncontrolled production animals. In such species the direct heritability of GL showed medium or high values for the low variability. In our opinion, the dromedary camel does not belong to the highly controlled production animals, nonetheless the CV\% of GL in our study showed a low value. This means that, contrary to our expectations, the variability of this trait was narrower than expected. So, the heritability of GL of camels in our study was medium.

Based on these findings, our initial hypothesis was partially confirmed. On the one hand, the direct heritability of GL was slightly higher and the maternal heritability of GL was lower than expected. On the other hand, the effect of environmental factors, based on previous results, especially those for the $c^{2}$, was in line with the expectations.

In conclusion, this study provides new data on genetic parameters of the GL trait using the world's largest dromedary camel dataset. Genetic parameters for the evaluated trait advanced our understanding of the interaction between genetic and environmental effects. To the best of our knowledge, BV estimates for any trait have not been published for the dromedary camel until now, with the exception of our previous work. The result that dromedary sires could be ranked according to their direct and maternal genetic effects, gave us the possibility to choose the sire most appropriate from this point of view to produce the next generation of dromedaries. All in all, the results obtained in this study provide new information that can help breeding programmes and sire selection in this species.

\section{ACKNOWLEDGEMENTS}

The authors would like to thank the management of Emirates Industry for Camel Milk and Products (EICMP, Dubai, UAE) for providing the facilities and supporting this research. We are also grateful to the staff of the company for monitoring deliveries, care of newborn camels and collection of data. The publication is supported by the EFOP-3.6.3VEKOP-16-2017-00008 project. The project is co-financed by the European Union and the European Social Fund.

\section{REFERENCES}

Abdallah, H. R. and Faye, B. (2012): Phenotypic classification of Saudi Arabian camel (Camelus dromedarius) by their body measurements. Emir. J. Food. Agric. 24, 272-280.

Agarwal, S. P., Khanna, N. D., Agarwal, V. K. and Dwarakanath, P. K. (1987): Circulating level of oestrogen and progesterone in female camels (Camelus dromedarius) during pregnancy. Theriogenology 28, 849-859.

Al-Bisher, B. E. (1998): Pregnancy and parturition in the camel (Camelus dromedarius) with particular reference to cervical dilatation. MVSc Thesis, King Faisal University, Saudi Arabia.

Al Eknah, M. M. (2000): Reproduction in old world camels. Anim. Reprod. Sci. 60-61, 583-592.

Almutairi, S. E., Boujenane, I., Musaad, A. and Awad-Acharari, F. (2010): Non-genetic factors influencing reproductive traits and calving weight in Saudi camels. Trop. Anim. Health Prod. 42, 1087-1092.

Alves, K., Schenkel, F. S., Brito, L. F. and Robinson, A. (2018): Estimation of direct and maternal genetic parameters for individual birth weight, weaning weight, and probe weight in Yorkshire and Landrace pigs. J. Anim. Sci. 96, 2567-2578.

Bene, Sz. (2013): Performance test results of stallions of different breeds between 1998-2010 in Hungary. 6th paper: Population genetics parameters, breeding values [in Hungarian, with English abstract]. Állattenyésztés és Takarmányozás [Hung. J. Anim. Prod.] 62, 21-36.

Bene, Sz., Benedek, Zs., Nagy, Sz., Szabó, F. and Polgár, J. P. (2014): Some effects on gestation length of traditional horse breeds in Hungary. J. Cent. Eur. Agric. 15, 1-10.

Bene, Sz., Szabó, F., Polgár, J. P., Juhász, J. and Nagy, P. (2020): Genetic parameters of birth weight trait in dromedary camels (Camelus dromedarius). Trop. Anim. Health Prod. 52, 23332340.

Boldman, K. G., Kriese, L. A., Van Vleck, L. D. and Kachman, S. D. (1993): A manual for use of MTDFREML. A set of programs to obtain estimates of variances and covariances. USDA-ARS, Clay Center, NE, USA.

Bos, H. and Van der Mey, G. J. W. (1980): Length of gestation periods of horses and ponies belonging to different breeds. Liv. Prod. Sci. 7, 181-187.

Bourdon, R. M. and Brinks, J. S. (1982): Genetic, environmental and phenotypic relationships among gestation length, birth weight, growth traits and age at first calving in beef cattle. J Anim. Sci. 55, 543-553.

Bouwman, A. C., Bergsma, R., Duijvesteijn, N. and Bijma, P. (2010): Maternal and social genetic effects on average daily gain of piglets from birth until weaning. J Anim. Sci. 88, 2883-2892.

Carnier, P., Albera, A., Dal Zotto, R., Groen, A. F., Bona, M. and Bittante, G. (2000): Genetic parameters for direct and maternal 
calving ability over parities in Piedmontese cattle. J Anim. Sci. 78, 2532-2539.

Christmann, A., Sieme, H., Martinsson, G. and Distl, O. (2017): Genetic and environmental factors influencing gestation length and parturition conception interval in Hanoverian warmblood. Livest. Sci. 199, 63-68.

Crews, D. H. (2006): Age of dam and sex of calf adjustments and genetic parameters for gestation length in Charolais cattle. J Anim. Sci. 84, 25-31.

Cubas, A. C., Berger, P. J. and Healey, M. H. (1991): Genetic parameters for calving ease and survival at birth in Angus field data. J. Anim. Sci. 69, 3952-3958.

Elias, E., Degen, A. A. and Kam, M. (1991): Effect of conception date on length of gestation in the dromedary camel (Camelus dromedarius) in the Negev Desert. Anim. Reprod. Sci. 25, 173-177.

Ewert, M., Lüders, I., Böröcz, J., Uphaus, H., Distl, O. and Sieme, H. (2018): Determinants of gestation length in Thoroughbred mares on German stud farms. Anim. Reprod. Sci. 191, 22-33.

Fábri, Zs. N. (2018): Endogenous and exogenous factors affecting the composition of raw camel's (Camelus dromedarius) milk [in Hungarian]. PhD dissertation, Széchenyi István University, Mosonmagyaróvár, Hungary. pp. 64-74.

Goyache, F., Gutiérrez, J. P., Fernández, I., Royo, L. J. and Álvarez, I. (2005): Genetic analysis of days open in beef cattle. Livest. Prod. Sci. 93, 283-289.

Hansen, M., Lund, M. S., Pedersen, J. and Christensen, L. G. (2004): Gestation length in Danish Holsteins has weak genetic associations with stillbirth, calving difficulty, and calf size. Livest. Prod. Sci. 91, 23-33.

Harvey, W. R. (1990): User's guide for LSLMW and MIXMDL PC2 version Mixed Model Least-Squares and Maximum Likelihood Computer Program. The Ohio State University, Columbus, OH, USA.

Haywood, L., Spike-Pierce, D. L., Barr, B., Mathys, D. and Mollenkopf, D. (2017): Gestation length and racing performance in 115 Thoroughbred foals with incomplete tarsal ossification. Equine Vet. J. 50, 29-33.

Heck, L., Clauss, M. and Sánchez-Villagra, M. R. (2018): Do domesticated mammals selected for intensive production have less variable gestation periods? Mamm. Biol. 88, 151-155.

Henderson, C. R. (1975): Best linear unbiased estimation and prediction under a selection model. Biometrics 31, 423-447.

Iwaisaki, H., Tsuruta, S., Misztal, I. and Bertrand, J. K. (2005): Estimation of correlation between maternal permanent environmental effects of related dams in beef cattle. J. Anim. Sci. 83, 537-542.

Langlois, B. and Blouin, C. (2012): Genetic parameters for gestation length in French horse breeds. Livest. Sci. 146, 133-139.

Lee, C., Van Tassel, C. P. and Pollak, E. J. (1997): Estimation of genetic variance and co-variance components for weaning weight in Simmental cattle. J. Anim. Sci. 75, 325-330.

Metha, V. S., Prakash, A. and Singh, M. (1962): Gestation period in camels. Indian Vet. J. 39, 387-389.

Meyer, K. (1998): DFREML. Version 3.0. User Notes.
Mujibi, F. D. N. and Crews, D. H. (2009): Genetic parameters for calving ease, gestation length, and birth weight in Charolais cattle. J. Anim. Sci. 87, 2759-2766.

Musa, E. E. and Abu Sineina, M. E. (1976): Some observations on reproduction in the female camel (Camelus dromedarius). Acta Vet. Beograd 26, 63-69.

Musa, H. H., Shuiep, E. S., Ibtisam, E., El Zubier, M. and Chen, G. H. (2006): Some reproductive and productive traits of camel (Camelus dromedarius) in Western Sudan. J. Anim. Vet. Adv. 5, 590-592.

Nagy, P. and Juhász, J. (2019): Pregnancy and parturition in dromedary camels I. Factors affecting gestation length, calf birth weight and timing of delivery. Theriogenology 134, 24-33.

Nagy, P., Skidmore, J. A. and Juhász, J. (2013): Use of assisted reproduction for the improvement of milk production in dairy camels (Camelus dromedarius). Anim. Reprod. Sci. 136, 205-210.

Novoa, C. (1970): Review: reproduction in Camelidae. J. Reprod. Fertil. 22, 3-20.

Pérez, C. C., Rodríguez, I., Mota, J., Dorado, J., Hidalgo, M., Felipe, M. and Sanz, J. (2003): Gestation length in Carthusian Spanishbred mares. Livest. Prod. Sci. 82, 181-187.

Phocas, F. and Laloë, D. (2004): Genetic parameters for birth and weaning traits in French specialized beef cattle breeds. Livest. Prod. Sci. 89, 121-128.

Ram, S., Singh, B. and Dhandha, O. P. (1977): A note on genetic studies on gestation length, birthweight and intrauterine development index in Indian camel (Camelus dromedarius) and factors affecting them. Indian Vet. J. 54, 953-955.

Rodrigues, J. A., Gonçalves, A. R., Antunes, L., Bettencourt, E. V. and Gama, L. T. (2020): Genetic and environmental factors influencing gestation length in Lusitano horses. J. Equine Vet. Sci. 84, 102850.

Sharma, S. S. and Vyas, K. K. (1971): Factors affecting gestation length in the Bikaneri camel (Camelus dromedarius). Ceylon Vet. J. 19, 67-68.

Szőke, Sz. and Komlósi, I. (2000): Comparison of BLUP models [in Hungarian, with English abstract]. Állattenyésztés és Takarmányozás [Hung. J. Anim. Prod.] 49, 231-246.

Trus, D. and Wilton, J. W. (1988): Genetic parameters for maternal traits in beef cattle. Can. J. Anim. Sci. 68, 119-128.

Valera, M., Blesa, F., Dos Santos, R. and Molina, A. (2006): Genetic study of gestation length in Andalusian and Arabian mares. Anim. Reprod. Sci. 95, 75-96.

Van Vleck, L. D., Gregory, K. E. and Bennett, G. L. (1996): Direct and maternal covariances by age of dam for weaning weight. J. Anim. Sci. 74, 1801-1805.

Willham, R. L. (1972): The role of maternal effects in animal breeding: III. Biometrical aspects of maternal effects in animals. J. Anim. Sci. 35, 1288-1293.

Wray, N. R., Quaas, R. L. and Pollak, E. J. (1987): Analysis of gestation length in American Simmental cattle. J. Anim. Sci. 65, 970-974.

Yagil, R. and Etzion, Z. (1984): Enhanced reproduction in camels (Camelus dromedarius). Comp. Biochem. Phys. A 79, 201-204.

Open Access. This is an open-access article distributed under the terms of the Creative Commons Attribution 4.0 International License (https://creativecommons.org/ licenses/by/4.0/), which permits unrestricted use, distribution, and reproduction in any medium, provided the original author and source are credited, a link to the CC License is provided, and changes - if any - are indicated. (SID_1) 